\title{
Modulatory effects of taurine on jejunal contractility
}

\author{
Q.Y. Yao, D.P. Chen, D.M. Ye, Y.P. Diao and Y. Lin \\ Dalian Medical University, Dalian, Liaoning, China
}

\begin{abstract}
Taurine (2-aminoethanesulfonic acid) is widely distributed in animal tissues and has diverse pharmacological effects. However, the role of taurine in modulating smooth muscle contractility is still controversial. We propose that taurine $(5-80 \mathrm{mM})$ can exert bidirectional modulation on the contractility of isolated rat jejunal segments. Different low and high contractile states were induced in isolated jejunal segments of rats to observe the effects of taurine and the associated mechanisms. Taurine induced stimulatory effects on the contractility of isolated rat jejunal segments at 3 different low contractile states, and inhibitory effects at 3 different high contractile states. Bidirectional modulation was not observed in the presence of verapamil or tetrodotoxin, suggesting that taurine-induced bidirectional modulation is $\mathrm{Ca}^{2+}$ dependent and requires the presence of the enteric nervous system. The stimulatory effects of taurine on the contractility of isolated jejunal segments was blocked by atropine but not by diphenhydramine or by cimetidine, suggesting that muscarinic-linked activation was involved in the stimulatory effects when isolated jejunal segments were in a low contractile state. The inhibitory effects of taurine on the contractility of isolated jejunal segments were blocked by propranolol and L-NG-nitroarginine but not by phentolamine, suggesting that adrenergic $\beta$ receptors and a nitric oxide relaxing mechanism were involved when isolated jejunal segments were in high contractile states. No bidirectional effects of taurine on myosin phosphorylation were observed. The contractile states of jejunal segments determine taurine-induced stimulatory or inhibitory effects, which are associated with muscarinic receptors and adrenergic $\beta$ receptors, and a nitric oxide associated relaxing mechanism.
\end{abstract}

Key words: Taurine; Bidirectional modulation; Enteric nervous system; Calcium dependent; Homeostasis; Intestinal motility

\section{Introduction}

Taurine (2-aminoethanesulfonic acid) is widely distributed in animal tissues, and plays an important role in diverse physiological processes including membrane stabilization (1), osmoregulation (2), regulation of cell apoptosis (3), antioxidation $(4,5)$, modulation of neuronal excitability $(6,7)$, and maintenance of $\mathrm{Ca}^{2+}$ homeostasis (8).

The function of taurine in the cardiovascular system has been well studied (9-11). Oral supplementation of taurine is shown to reduce blood pressure and improve cardiac function in hypertensive subjects (12-14). Ex vivo studies have shown that contractile responses to norepinephrine $(\mathrm{NE})$ and $\mathrm{KCl}$ in aortic rings are attenuated both in taurinetreated normal rats and diabetic rats compared with controls $(15,16)$; NE-, $\mathrm{KCl}$-, and adenosine-receptor-agonistinduced hypercontractility of the aorta are enhanced in taurine-depleted rats compared with the effects in control animals $(17,18)$. Although most studies show that taurine induces inhibitory effects in precontracted vessels, some reports indicate that taurine at concentrations of $20-60 \mathrm{mM}$ inhibits phenylephrine-induced contraction in normal arteries of rats without affecting the basal tone of the arteries (19). However, taurine further enhances the NE- or $\mathrm{KCl}$-induced contraction of arteries in the insulin-resistant rat (20). The studies cited above indicate that the effects of taurine on vascular smooth muscle are still controversial and the associated mechanisms remain unclear.

The divergent effects of taurine on vascular smooth muscle attracted our interest. We proposed a bidirectional modulation of taurine on smooth muscle and noted that the effects of taurine on intestinal smooth muscle contraction are rarely reported. It is known that the contraction of intestinal smooth muscle is modulated by the enteric nervous system (ENS), which can control functions of the intestine even when it is completely separated from the central nervous system (CNS) (21). To characterize the effects of taurine on intestinal contractility and reveal the possible mechanism, isolated jejunal segments and 3 different pairs of low and high contractile states of the segments were established by modification of ionic concentrations or by inhibitory and stimulatory neurotransmitters. 

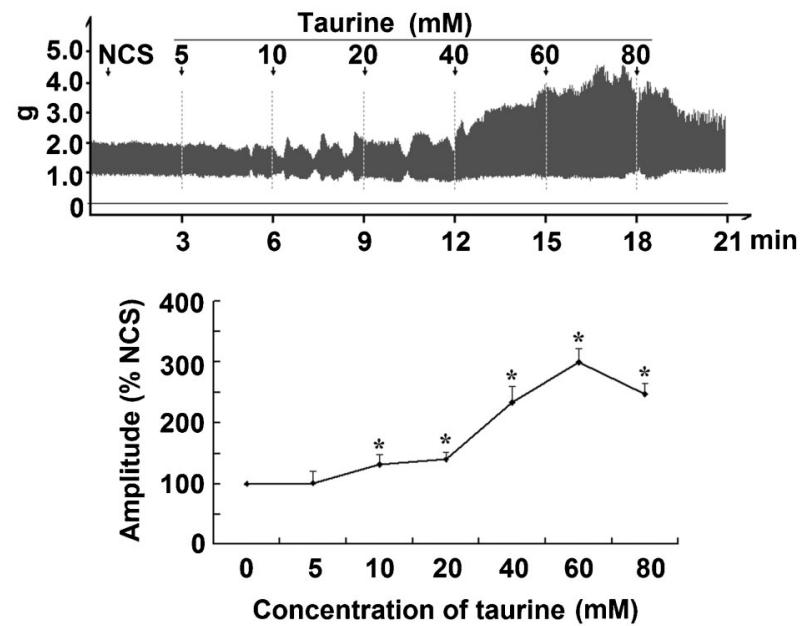

Figure 1. Effects of taurine on the contractile amplitude of isolated jejunal segments. Representative traces and statistical analysis $(n=6)$ of taurine-induced effects on the contractile amplitude of isolated jejunal segments in the normal contractile state (NCS, control). The contractile amplitude in NCS is set to $100 \%$; the effects of taurine on the contractile amplitude are the relative values compared with NCS. ${ }^{*} \mathrm{P}<0.05$ vs the contractile amplitude in NCS before taurine administration (one-way ANOVA).
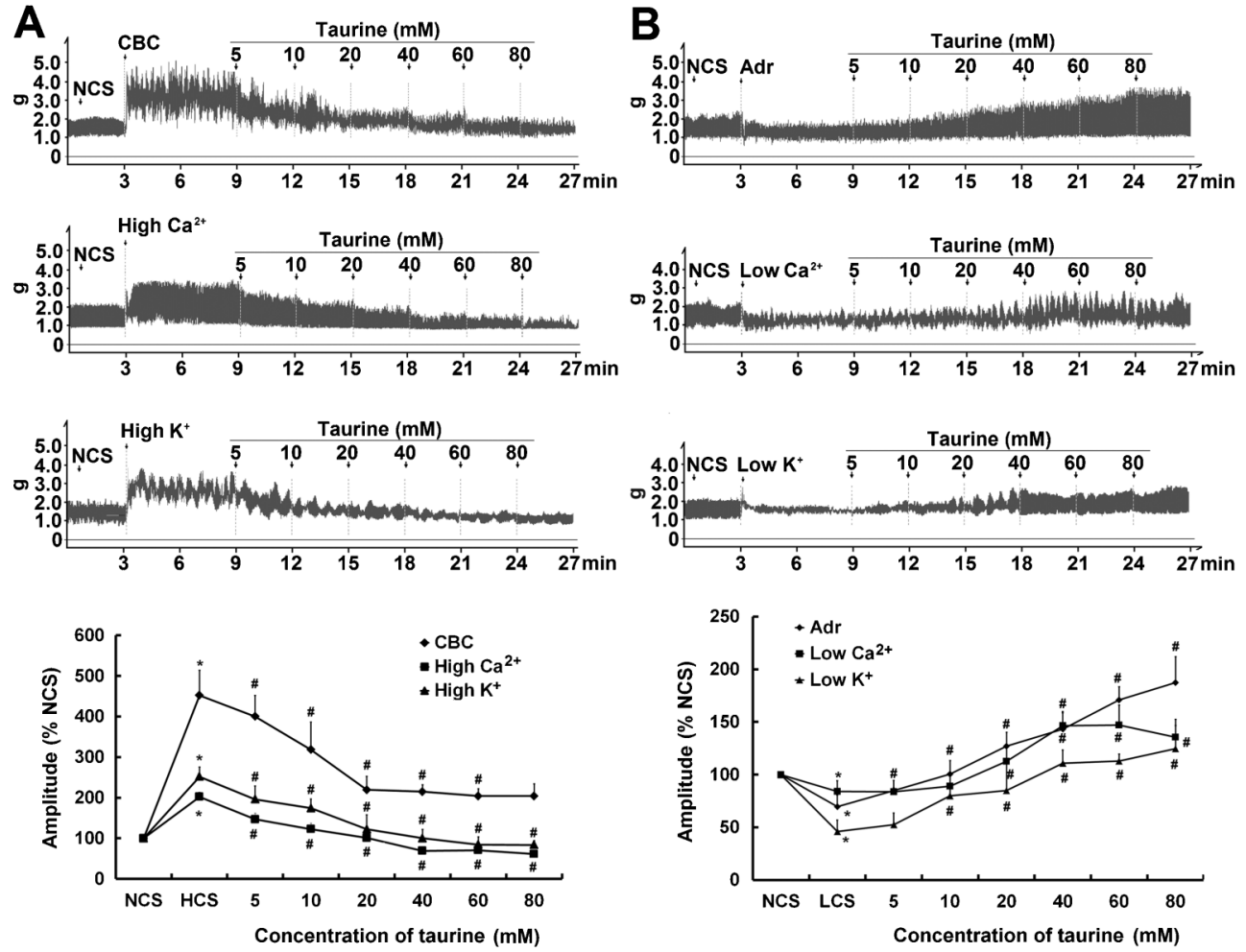

Figure 2. Taurine-induced bidirectional modulations on the contractile amplitude of isolated jejunal segments. $A$, Representative traces and statistical analysis $(n=6)$ of the inhibitory effects of taurine on the contractile amplitude of isolated jejunal segments in 3 high contractile states (HCS). B, Representative traces and statistical analysis $(n=6)$ of the stimulatory effects of taurine on the contractile amplitude of isolated jejunal segments in 3 low contractile states (LCS). The contractile amplitude in the normal contractile state (NCS, control) is set to $100 \%$; the contractile amplitude in HCS and LCS are the relative values compared with NCS. CBC: carbachol; Adr: adrenaline. ${ }^{*} P<0.05$ vs the contractile amplitude in NCS; ${ }^{\#} \mathrm{P}<0.05$ vs the contractile amplitude in LCS or HCS before taurine administration (one-way ANOVA). 

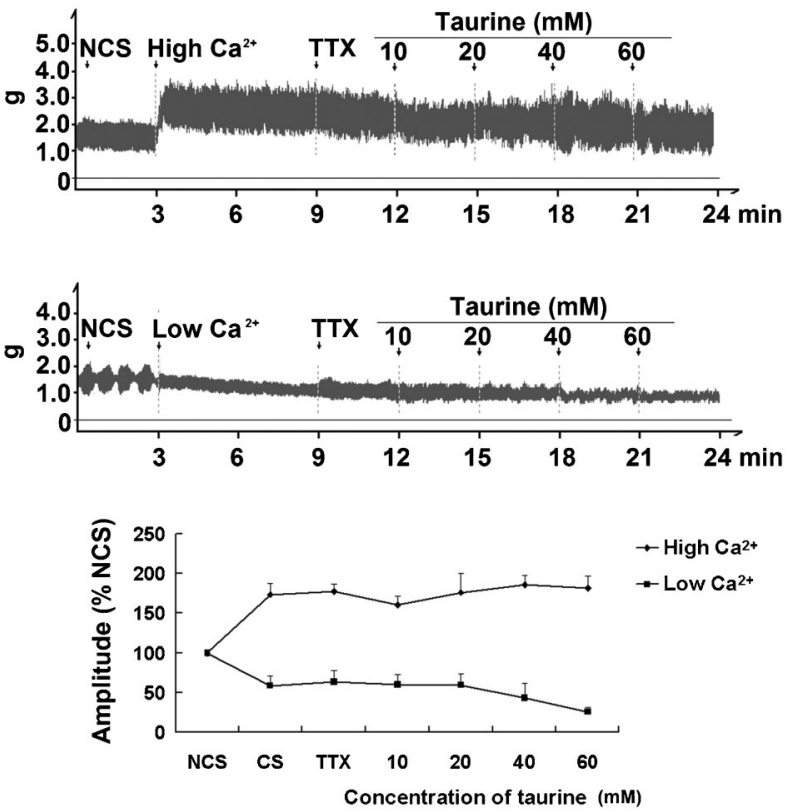

Figure 3. Effects of taurine on the contractile amplitude of isolated jejunal segments pretreated with tetrodotoxin (TTX). Representative traces and statistical analysis $(n=6)$ of taurineinduced effects on the contractile amplitude of isolated jejunal segments in the normal contractile state (NCS, control), high contractile state $(\mathrm{HCS})$ induced by high $\mathrm{Ca}^{2+}(5.0 \mathrm{mM})$ Krebs buffer and low contractile state (LCS) induced by low $\mathrm{Ca}^{2+}$ $(1.25 \mathrm{mM})$ Krebs buffer pretreated with TTX $(0.3 \mu \mathrm{M})$. The contractile amplitude in NCS is set to $100 \%$; other data are the relative values compared with NCS. CS: contractile state.

$\mathrm{MgSO}_{4}, 4.2 \mathrm{mM} \mathrm{NaHCO}_{3}, 2.5 \mathrm{mM} \mathrm{CaCl}_{2}, 10 \mathrm{mM}$ glucose, $\mathrm{pH}$ 7.4). The isolated jejunum was rinsed, clipped into segments (about $12 \mathrm{~mm}$ long), and the mesenteric border was removed. The segments were suspended in longitudinal orientation in $20-\mathrm{mL}$ organ baths of warm $\left(37^{\circ} \mathrm{C}\right)$, aerated Krebs buffer. The distal end of each segment was tied to an organ holder, and the proximal end was secured with a silk thread to an isometric force transducer. A $1-\mathrm{g} / \mathrm{cm}$ resting tension was applied to each segment, which was equilibrated for about $1 \mathrm{~h}$. Rhythmic spontaneous contractions developed in all preparations. The contractile responses of the isolated jejunal segment were recorded with a BL-420 physiological recording system (Chengdu Taimeng Software Co., Ltd., China).

The contractility of isolated jejunal segments in Krebs buffer was selected as the normal contractile state (NCS). Modified low-Ca ${ }^{2+}(1.25 \mathrm{mM})$, low $\mathrm{K}^{+}(2.5 \mathrm{mM})$, or adrenaline $(5 \mu \mathrm{M})$ Krebs buffers were selected as low contractile states (LCS). Modified high- $\mathrm{Ca}^{2+}(5.0 \mathrm{mM})$, high- $\mathrm{K}^{+}$ (10.0 mM), or carbachol (CBC, $1 \mu \mathrm{M})$ Krebs buffers were selected as high contractile states (HCS). To study the mechanisms of taurine-induced bidirectional modulation, the HCS induced by high $\mathrm{Ca}^{2+}(5.0 \mathrm{mM})$ Krebs buffer and LCS induced by low $\mathrm{Ca}^{2+}(1.25 \mathrm{mM})$ Krebs buffer were selected as the representative HCS and representative LCS, respectively. A sodium channel blocker (TTX, $0.3 \mu \mathrm{M}$ for $3 \mathrm{~min}$ ); a voltage-dependent L-type calcium channel blocker (verapamil, $1 \mu \mathrm{M}$ for $3 \mathrm{~min}$ ); an adrenergic $\alpha$ receptor antagonist (phentolamine, $1 \mu \mathrm{M}$ for $3 \mathrm{~min}$ ); an adrenergic $\beta$-receptor antagonist (propranolol, $1 \mu \mathrm{M}$ for $3 \mathrm{~min}$ ); a cholinergic $\mathrm{M}$ receptor antagonist (atropine, $1 \mu \mathrm{M}$ for $3 \mathrm{~min}$ ); a histamine $\mathrm{H}_{1}$-receptor antagonist (diphenhydramine, $10 \mu \mathrm{M}$ for $3 \mathrm{~min}$ ); a histamine $\mathrm{H}_{2}$-receptor antagonist (cimetidine, $10 \mu \mathrm{M}$ for $3 \mathrm{~min}$ ), and a nitric oxide (NO) synthase inhibitor $\left[\mathrm{L}-\mathrm{N}^{\mathrm{G}}\right.$-nitroarginine (L-NNA), $300 \mu \mathrm{M}$ for $3 \mathrm{~min}$ ] were used in the assay. The contractile amplitude of isolated jejunal segments is reported as a percentage of the contractile amplitude in the NCS. The contractile amplitude at NCS was set to a relative value of $100 \%$.

\section{Measurement of myosin phosphorylation}

Myosin and myosin light chain kinase (MLCK) used in the assay were purified from chicken gizzard smooth muscle as described previously (22). Myosin phosphorylation was carried out in a $20 \mathrm{mM}$ Tris- $\mathrm{HCl}, \mathrm{pH} 7.4$, buffer containing $1 \mathrm{mM}$ dithiothreitol (DTT), $5 \mathrm{mM} \mathrm{MgCl}_{2}, 60 \mathrm{mM}$ $\mathrm{KCl}, 0.1 \mathrm{mM} \mathrm{CaCl}_{2}, 0.6 \mu \mathrm{M}$ calmodulin, $4 \mu \mathrm{M}$ myosin, and $2 \mathrm{mM}$ ATP with or without taurine at $25^{\circ} \mathrm{C}$ for $20 \mathrm{~min}$. MLCK (2.0 and $0.02 \mu \mathrm{M}$ ) was used to phosphorylate $\mathrm{MLC}_{20}$, exerting a high-extent and low-extent of phosphorylation, respectively. The extent of $20-\mathrm{kDa}$ myosin light chain $\left(\mathrm{MLC}_{20}\right)$ phosphorylation was measured by $10 \%$ glycerol polyacrylamide gel electrophoresis (PAGE) $(23,24)$. A GelPro Analyzer (Media Cybernetics, USA) was used to scan the density and size of phosphorylated $\mathrm{MLC}_{20}$. The extent of phosphorylated $\mathrm{MLC}_{20}$ is reported as the percentage of phosphorylated $\mathrm{MLC}_{20}$ in total $\mathrm{MLC}_{20}$. The extent of full mono-phosphorylation was set at $100 \%$ (control).

\section{Statistical analysis}

All data are reported as means \pm SE. Statistical significance was tested by one-way analysis of variance, followed by the Dunnett multiple comparisons test, using the SPSS software 13.0 (SPSS Inc., USA). Statistical significance was defined as $\mathrm{P}<0.05$.

\section{Results}

\section{Effects of taurine on the contractility of isolated jejunal segments}

Taurine (5-80 mM) enhanced the contractile amplitude of isolated jejunal segments in a concentration-dependent manner (Figure 1, Figure S1). The contractile amplitude in both HCS and LCS was statistically different from that of NCS (Figure 2, Figure S2). Taurine (5-80 mM) significantly enhanced the contractile amplitude of jejunal segments in all 3 LCS $(\mathrm{P}<0.05)$, and significantly decreased the amplitude in all $3 \mathrm{HCS}(\mathrm{P}<0.05$, Figure 2, Figure S2). These data indicated that taurine induced bidirectional modulation of the 

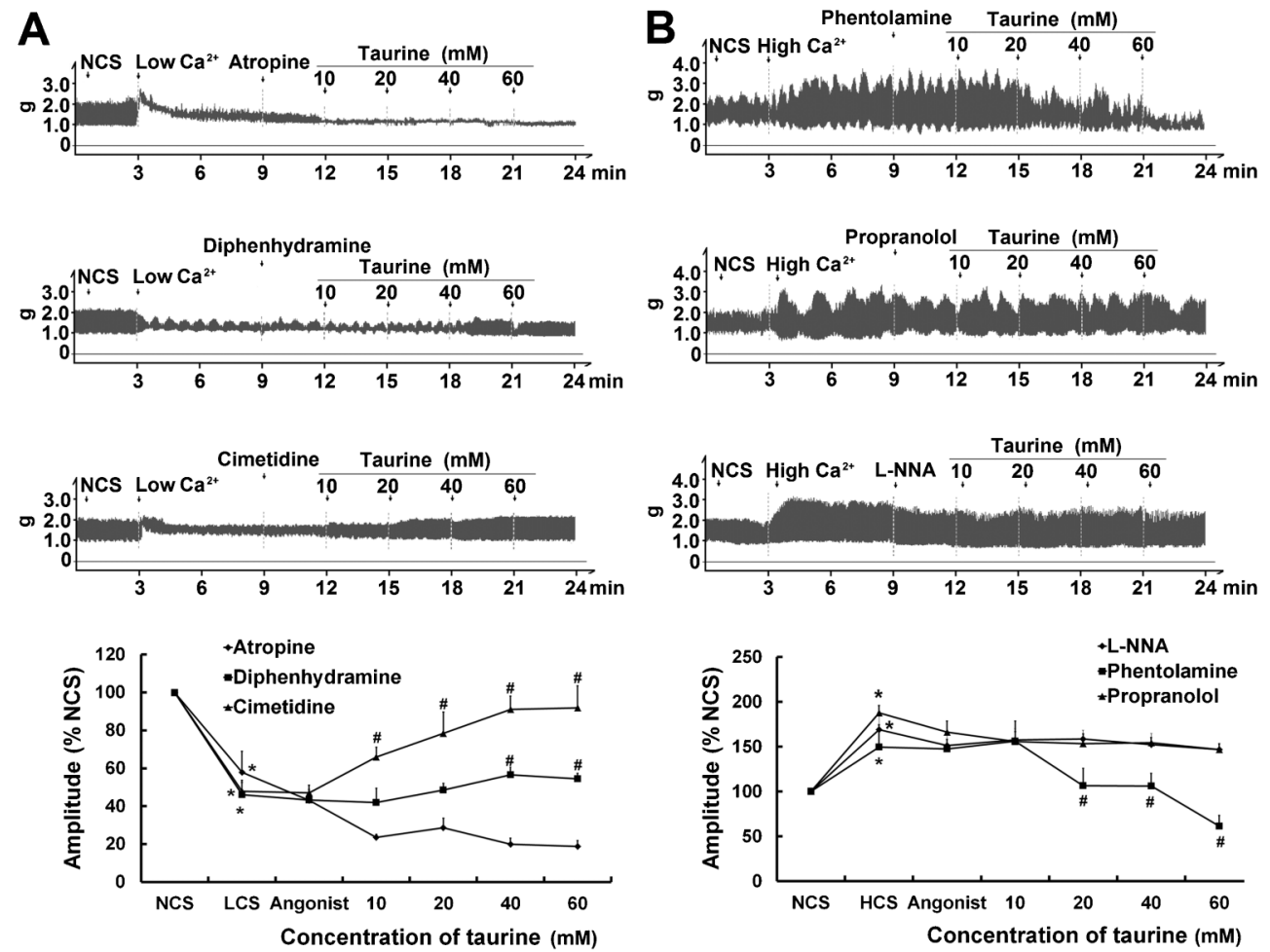

Figure 4. Effects of taurine on the contractile amplitude of isolated jejunal segments pretreated with receptor antagonists. $A$, Representative traces and statistical analysis $(n=6)$ of taurine-induced effects on the contractile amplitude of isolated jejunal segments pretreated with $1 \mu \mathrm{M}$ atropine, $10 \mu \mathrm{M}$ diphenhydramine, and $10 \mu \mathrm{M}$ cimetidine in low contractile states (LCS) induced by low Ca ${ }^{2+}$ $(1.25 \mathrm{mM})$ Krebs buffer. $B$, Representative traces and statistical analysis $(n=6)$ of taurine-induced effects on the contractile amplitude of isolated jejunal segments pretreated with $1 \mu \mathrm{M}$ phentolamine, $1 \mu \mathrm{M}$ propranolol, and $300 \mu \mathrm{M} \mathrm{L}-\mathrm{NNA}$ in high contractile states (HCS) induced by high $\mathrm{Ca}^{2+}(5.0 \mathrm{mM})$ Krebs buffer. The contractile amplitude in the normal contractile state (NCS, control) is set to $100 \%$; other data are the relative values compared with NCS. ${ }^{*} \mathrm{P}<0.05$ vs the contractile amplitude in $\mathrm{NCS} ;{ }^{\#} \mathrm{P}<0.05$ vs the contractile amplitude in LCS or HCS before taurine administration (one-way ANOVA).

spontaneous contractility of isolated jejunal segments.

\section{Underlying mechanism of taurine-induced bidirectional modulation}

In the presence of TTX, neither an inhibitory effect of taurine $(10-60 \mathrm{mM})$ on the contractile amplitude of isolated jejunal segments in the HCS induced by high $\mathrm{Ca}^{2+}(5.0 \mathrm{mM})$ Krebs buffer nor a stimulatory effect on the contractile amplitude in LCS induced by low $\mathrm{Ca}^{2+}(1.25 \mathrm{mM})$ Krebs buffer were observed (Figure 3 ). These data showed that TTX abolished bidirectional modulation of taurine on jejunal contractility.

The nonselective muscarinic receptor antagonist atropine blocked the stimulatory effect of taurine $(10-60 \mathrm{mM})$ on the contractile amplitude of isolated jejunal segments in LCS induced by low $\mathrm{Ca}^{2+}(1.25 \mathrm{mM})$ Krebs buffer. Neither the histamine $\mathrm{H}_{1}$-receptor antagonist diphenhydramine nor the $\mathrm{H}_{2}$-receptor antagonist cimetidine blocked taurine (10-60 mM)-induced stimulatory effects on the contractile amplitude in the LCS induced by low $\mathrm{Ca}^{2+}(1.25 \mathrm{mM})$ Krebs buffer (Figure 4). In the HCS induced by high $\mathrm{Ca}^{2+}$
(5.0 mM) Krebs buffer, $\beta$-adrenergic receptor antagonist propranolol, and NO synthase inhibitor L-NNA blocked the taurine (10-60 mM)-induced inhibitory effect on the contractile amplitude of isolated jejunal segments. However, the $\alpha$-adrenergic receptor antagonist phentolamine did not block the taurine-induced (10-60 mM) inhibitory effects on the contractile amplitude in the HCS induced by high $\mathrm{Ca}^{2+}$ (5.0 mM) Krebs buffer (Figure 4).

Pre-incubation of isolated jejunal segments with the $\mathrm{Ca}^{2+}$ channel blocker verapamil at NCS, HCS induced by high $\mathrm{Ca}^{2+}(5.0 \mathrm{mM})$ Krebs buffer, and LCS induced by low $\mathrm{Ca}^{2+}(1.25 \mathrm{mM})$ Krebs buffer abolished the effects of taurine $(10-60 \mathrm{mM})$ on the contractile amplitude of isolated jejunal segments (Figure 5). This suggests that verapamil blocked the effects of taurine-induced contractility of isolated jejunal segments.

No significant effects of taurine on myosin phosphorylation were observed at concentrations of $5-40 \mathrm{mM}$. Although the effects of $80 \mathrm{mM}$ taurine were significant, both high-extent and low-extent of myosin phosphorylation were affected (Figure 6). These data indicate that taurine 

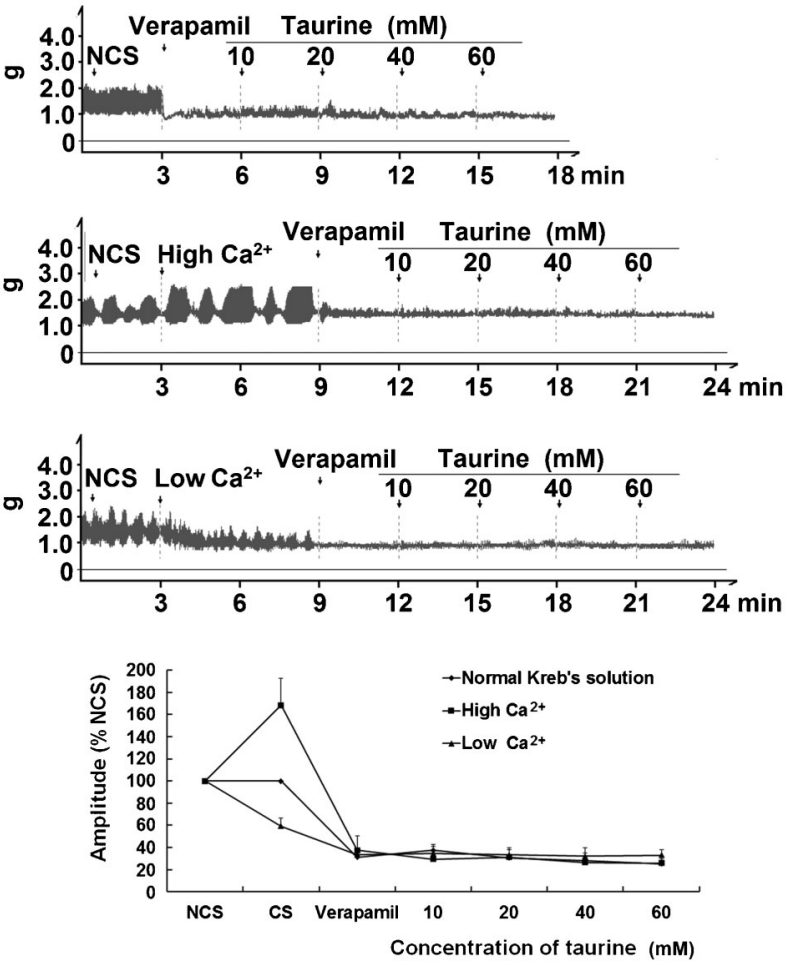

Figure 5. Effects of taurine on the contractile amplitude of isolated jejunal segments pretreated with verapamil. Representative traces and statistical analysis $(n=6)$ of taurine-induced effects on the contractile amplitude of isolated jejunal segments pretreated with $1 \mu \mathrm{M}$ verapamil in the normal contractile state (NCS, control), CS induced by high $\mathrm{Ca}^{2+}(5.0 \mathrm{mM})$ Krebs buffer and CS induced by low $\mathrm{Ca}^{2+}(1.25 \mathrm{mM})$ Krebs buffer. The mean contractile amplitude without verapamil treatment in NCS is set to $100 \%$; other data are the relative values compared with NCS. CS: contractile state.

did not modulate myosin phosphorylation bidirectionally.

\section{Discussion}

Taurine exerted stimulatory and inhibitory effects on the contractility of isolated jejunal segments in both low and high contractile states. Considering that the HCS and LCS were established under different assay conditions and by using agents with different mechanisms, the results suggest that taurine-induced bidirectional modulation on isolated rat jejunal segments depends on its contractile state. The evidence that taurine induced a bidirectional modulation on jejunal contractility depending on its contractile state indicates that taurine plays an important homeostatic role in intestinal function.

$\mathrm{Ca}^{2+} / \mathrm{CaM}-$ dependent phosphorylation of myosin light chains by MLCK is generally considered to be the primary mechanism for regulating the contraction of smooth muscle. Nevertheless, the effects of taurine on phosphorylation of myosin from chicken gizzard were not consistent with its

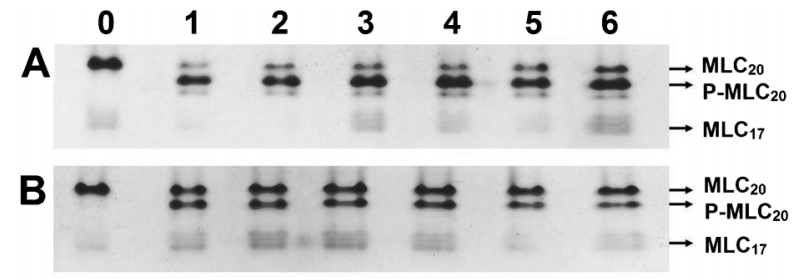

C

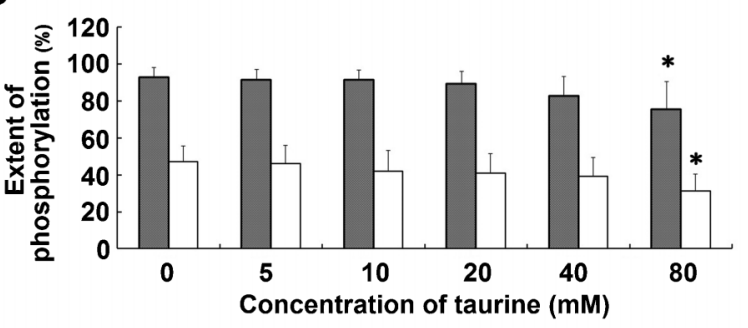

- High-extent phosphorylated myosin

$\checkmark$ Low-extent phosphorylated myosin

Figure 6. Effects of taurine on phosphorylated myosin. A, Effects of taurine on high-extent phosphorylated myosin, $0.02 \mu \mathrm{M}$ myosin light chain kinase (MLCK), and $4.0 \mu \mathrm{M}$ myosin purified from chicken gizzard smooth muscle used in the assay. $B$, Effects of taurine on low-extent phosphorylated myosin, $2.0 \mu \mathrm{M}$ MLCK and $4.0 \mu \mathrm{M}$ myosin purified from chicken gizzard smooth muscle used in the assay. Lanes 0-6 represent unphosphorylated myosin (without MLCK and taurine), high- or low-extent phosphorylated control (without taurine), high- or low-extent phosphorylated myosin with $5,10,20,40$, and $80 \mathrm{mM}$ taurine, respectively. C, Extent of myosin phosphorylation, which was analyzed using the Gelpro software. The extent of phosphorylated myosin was calculated as percentage of phosphorylated regulatory myosin light chain of $20 \mathrm{kDa}\left(\mathrm{MLC}_{20}\right)$ in total $\mathrm{MLC}_{20}$. Mono-phosphorylation was calculated as $100 \%$ phosphorylation. ${ }^{*} \mathrm{P}<0.05$ vs highor low-extent phosphorylated control (without taurine) (one-way ANOVA) (lane 1). $\mathrm{MLC}_{20}$ : unphosphorylated $\mathrm{MLC}_{20}$ (20 kDa regulatory myosin light chain); $\mathrm{p}-\mathrm{MLC}_{20}$ : mono-phosphorylated $\mathrm{MLC}_{20} ; \mathrm{MLC}_{17}$ : $17 \mathrm{kDa}$ myosin essential light chains.

bidirectional effect on isolated segments of rat jejunum. We only observed a slight inhibition effect of taurine on both high- and low-extent phosphorylation of myosin from chicken gizzard. Considering that such a high intracellular concentration of taurine can hardly be achieved in the muscle, we believe that the inhibition of $80 \mathrm{mM}$ taurine on myosin phosphorylation was due to the toxic effects induced by such high concentration. These results indicated that taurine might exert dual modulation not on myogenic contractions of intestinal smooth muscle directly, but by some other mechanisms, e.g., regulation of neurotransmitter or hormone release. However, due to species differences, we cannot completely exclude the possibility that taurine has a direct effect on phosphorylation of myosin light chains in rats.

The neuronal regulation of intestinal contraction involves the ENS, as well as extrinsic nerves (25); TTX is a blocker of neuronal conduction (26); taurine-induced modulation on 
the contractility of isolated jejunal segments was blocked by TTX, suggesting that neural regulation of ENS is involved in taurine-induced effects.

Activation of muscarinic or histamine receptor increases intestinal contractility, and stimulation of $\alpha$ and $\beta$ adrenoceptors inhibits intestinal contractility. Inhibition of intestinal contractility is also mediated by $\mathrm{NO}$, a nonadrenergic, noncholinergic neurotransmitter that produces its effect by directly acting on smooth muscle and by indirectly inhibiting acetylcholine and substance $P$ release $(27,28)$. In LCS induced by low $\mathrm{Ca}^{2+}(1.25 \mathrm{mM})$ Krebs buffer, atropine blocked the stimulatory effects of taurine on the contractility of isolated jejunal segments, but diphenhydramine and cimetidine did not, implicating that the stimulatory effects of taurine on the jejunal contractility are correlated with muscarinic receptor-linked stimulation. The results are in line with reports that found the effect of taurine $\left(10^{-6}-10^{-1} \mathrm{mM}\right)$ on acid secretion in the rat stomach was completely or partially inhibited by atropine (29). Although the doses of taurine used in our study were different from those in the above report, the difference might be due to the different target tissues of taurine. In HCS induced by high $\mathrm{Ca}^{2+}(5.0 \mathrm{mM})$ Krebs buffer, propranolol and L-NNA abolished the inhibitory effects of taurine on the contractility of isolated jejunal segments, however, phentolamine did not modify the inhibitory effects of taurine, suggesting that the inhibitory effects of taurine are correlated with the activation of adrenergic $\beta$ receptors, as well as NO synthase-linked relaxation mechanisms, rather than adrenergic $\alpha$ receptors. Consistent with our results, taurine has been reported to increase serum levels of $\mathrm{NO}, \mathrm{NO}$ synthase, and reactive nitrogen oxide species in the rat model of hypertension (30) and in guinea pig spleen tissue (4). However, the possible mechanisms of taurine on NO synthase in our study and the above studies may differ,

\section{References}

1. Junyent $F$, de Lemos L, Utrera J, Paco S, Aguado F, Camins $A$, et al. Content and traffic of taurine in hippocampal reactive astrocytes. Hippocampus 2011; 21: 185-197, doi: 10.1002/ hipo.20739.

2. Morales I, Dopico JG, Sabate M, Gonzalez-Hernandez T, Rodriguez M. Substantia nigra osmoregulation: taurine and ATP involvement. Am J Physiol Cell Physiol 2007; 292: C1934-C1941, doi: 10.1152/ajpcell.00593.2006.

3. Zhang LY, Zhou YY, Chen F, Wang B, Li J, Deng YW, et al. Taurine inhibits serum deprivation-induced osteoblast apoptosis via the taurine transporter/ERK signaling pathway. Braz J Med Biol Res 2011; 44: 618-623, doi: 10.1590/ S0100-879X2011007500078.

4. Bircan FS, Balabanli B, Turkozkan N, Ozan G. Effects of taurine on nitric oxide and 3-nitrotyrosine levels in spleen during endotoxemia. Neurochem Res 2011; 36: 1978-1983, doi: 10.1007/s11064-011-0521-3.

5. Silva LA, Silveira PC, Ronsani MM, Souza PS, Scheffer D, Vieira LC, et al. Taurine supplementation decreases oxidative stress in skeletal muscle after eccentric exercise. and remains uncertain in this study

Smooth muscle contraction is initiated by an increase in cytosolic free $\mathrm{Ca}^{2+}$, brought about either by release of $\mathrm{Ca}^{2+}$ from intracellular stores or by influx of $\mathrm{Ca}^{2+}$ through voltage-dependent $\mathrm{Ca}^{2+}$ channels (31). Our results indicated that verapamil, an L-type $\mathrm{Ca}^{2+}$ channel antagonist, blocked the effect of taurine on the contractility of isolated jejunal segments in NCS and HCS induced by high $\mathrm{Ca}^{2+}(5.0 \mathrm{mM})$ Krebs buffer and LCS induced by low $\mathrm{Ca}^{2+}$ $(1.25 \mathrm{mM})$ Krebs buffer, suggesting that modulation of intestinal contractility by taurine is $\mathrm{Ca}^{2+}$ dependent and involves L-type $\mathrm{Ca}^{2+}$ channels. It has been demonstrated that taurine may exert a normalizing action on the $\left[\mathrm{Ca}^{2+}\right]_{i}$ by directly or indirectly modulating the activity of the voltage-dependent $\mathrm{Ca}^{2+}$ channels or other transmembrane ion channels and transporters in the cell organelles $(16,32-34)$. Such dual effects on $\left[\mathrm{Ca}^{2+}\right]_{i}$ may explain the bidirectional modulation of taurine on smooth muscle contraction.

Although the present research characterized a homeostatic modulation of taurine on contractility of intestinal smooth muscle, it should still be considered as preliminary. Further studies are needed to reveal the detailed mechanism, including the possible neurotransmitters involved and the details of cell signal transduction.

\section{Supplementary Material}

Click here to view [pdf]

\section{Acknowledgments}

Research supported by the National Natural Science Foundation of China (No. 30772601).

Cell Biochem Funct 2011; 29: 43-49, doi: 10.1002/cbf.1716.

6. Gao X, Yang X, Zhang B. Neuroprotection of taurine against bilirubin-induced elevation of apoptosis and intracellular free calcium ion in vivo. Toxicol Mech Methods 2011; 21: 383387, doi: 10.3109/15376516.2010.546815.

7. Haojun Z, Yaoling W, Ke Z, Jin L, Junling W. Effects of NaF on the expression of intracellular $\mathrm{Ca}^{2+}$ fluxes and apoptosis and the antagonism of taurine in murine neuron. Toxicol Mech Methods 2012; 22: 305-308, doi: 10.3109/15376516.2012. 657259.

8. Huxtable RJ. Physiological actions of taurine. Physiol Rev 1992; 72: 101-163.

9. Wojcik OP, Koenig KL, Zeleniuch-Jacquotte A, Costa M, Chen $Y$. The potential protective effects of taurine on coronary heart disease. Atherosclerosis 2010; 208: 19-25, doi: 10.1016/j. atherosclerosis.2009.06.002.

10. Abebe W, Mozaffari MS. Role of taurine in the vasculature: an overview of experimental and human studies. Am J Cardiovasc Dis 2011; 1: 293-311.

11. Souza MM, Boyle RT. A moderate decrease in temperature 
inhibits the calcium signaling mechanism(s) of the regulatory volume decrease in chick embryo cardiomyocytes. Braz $J$ Med Biol Res 2001; 34: 137-141, doi: 10.1590/S0100879X2001000100018.

12. Ideishi M, Miura S, Sakai T, Sasaguri M, Misumi Y, Arakawa $\mathrm{K}$. Taurine amplifies renal kallikrein and prevents saltinduced hypertension in Dahl rats. J Hypertens 1994; 12: 653-661, doi: 10.1097/00004872-199406000-00005.

13. Anuradha CV, Balakrishnan SD. Taurine attenuates hypertension and improves insulin sensitivity in the fructose-fed rat, an animal model of insulin resistance. Can J Physiol Pharmacol 1999; 77: 749-754, doi: 10.1139/y99-060.

14. Militante JD, Lombardini JB. Treatment of hypertension with oral taurine: experimental and clinical studies. Amino Acids 2002; 23: 381-393, doi: 10.1007/s00726-002-0212-0.

15. Abebe W, Mozaffari MS. Effects of chronic taurine treatment on reactivity of the rat aorta. Amino Acids 2000; 19: 615623, doi: 10.1007/s007260070011.

16. Abebe W. Effects of taurine on the reactivity of aortas from diabetic rats. Life Sci 2008; 82: 279-289, doi: 10.1016/j.lfs. 2007.11.012.

17. Abebe W, Mozaffari MS. Effect of taurine deficiency on adenosine receptor-mediated relaxation of the rat aorta. Vascul Pharmacol 2003; 40: 219-228, doi: 10.1016/j.vph. 2003.08.001.

18. Abebe W, Mozaffari MS. Taurine depletion alters vascular reactivity in rats. Can J Physiol Pharmacol 2003; 81: 903909, doi: 10.1139/y03-088.

19. Niu LG, Zhang MS, Liu Y, Xue WX, Liu DB, Zhang J, et al. Vasorelaxant effect of taurine is diminished by tetraethylammonium in rat isolated arteries. Eur J Pharmacol 2008; 580: 169-174, doi: 10.1016/j.ejphar.2007.10.039.

20. Xue W, Zhang M, Li J, Wu D, Niu L, Liang Y. Effects of taurine on aortic rings isolated from fructose-fed insulin resistance Sprague-Dawley rat are changed. Cardiovasc Drugs Ther 2008; 22: 461-468, doi: 10.1007/s10557-008-6124-9.

21. Schemann M. Control of gastrointestinal motility by the "gut brain" - the enteric nervous system. J Pediatr Gastroenterol Nutr 2005; 41 (Suppl 1): S4-S6, doi: 10.1097/01.scs.000018 0285.51365.55.

22. Lin $\mathrm{Y}$, Ishikawa $\mathrm{R}$, Okagaki $\mathrm{T}, \mathrm{Ye} \mathrm{LH}$, Kohama $\mathrm{K}$. Stimulation of the ATP-dependent interaction between actin and myosin by a myosin-binding fragment of smooth muscle caldesmon. Cell Motil Cytoskeleton 1994; 29: 250-258, doi: 10.1002/cm.970290308.
23. Lin $\mathrm{Y}$, Sun H, Dai S, Tang Z, He X, Chen H. The bi-directional regulation of filamin on the ATPase activity of smooth muscle myosin. Chin Med Sci J 2000; 15: 162-164.

24. Yang J, Wang $X$, Tang $Z$, Chen $H$, Dai S, Lin $Y$. The characterization of myosin light chain phosphorylation by the constitutively active fragment of MLCK. Chinese Med Sci J (Chung-kuo i hsueh k'o hsueh tsa chih) 2003; 18: 206212, http://www.cqvip.com/Main/Detail.aspx?id=9051177.

25. Hansen MB. Neurohumoral control of gastrointestinal motility. Physiol Res 2003; 52: 1-30.

26. Llewellyn LE. Sodium channel inhibiting marine toxins. Prog Mol Subcell Biol 2009; 46: 67-97, doi: 10.1007/978-3-54087895-7_3.

27. Li M, Johnson CP, Adams MB, Sarna SK. Cholinergic and nitrergic regulation of in vivo giant migrating contractions in rat colon. Am J Physiol Gastrointest Liver Physiol 2002; 283: G544-G552.

28. Mang CF, Truempler S, Erbelding $\mathrm{D}$, Kilbinger $\mathrm{H}$. Modulation by NO of acetylcholine release in the ileum of wild-type and NOS gene knockout mice. Am J Physiol Gastrointest Liver Physiol 2002; 283: G1132-G1138.

29. Huang KH, Chang CC, Ho JD, Lu RH, Tsai LH. Role of taurine on acid secretion in the rat stomach. J Biomed Sci 2011; 18: 11, doi: 10.1186/1423-0127-18-11.

30. Hu J, Xu X, Yang J, Wu G, Sun C, Lv Q. Antihypertensive effect of taurine in rat. Adv Exp Med Biol 2009; 643: 75-84, doi: 10.1007/978-0-387-75681-3_8.

31. Aviello G, Scalisi C, Fileccia R, Capasso R, Romano B, Izzo $A A$, et al. Inhibitory effect of caffeic acid phenethyl ester, a plant-derived polyphenolic compound, on rat intestinal contractility. Eur J Pharmacol 2010; 640: 163-167, doi: 10.1016/ j.ejphar.2010.04.040.

32. Sawamura A, Sada H, Azuma J, Kishimoto S, Sperelakis N Taurine modulates ion influx through cardiac $\mathrm{Ca}^{2+}$ channels. Cell Calcium 1990; 11: 251-259, doi: 10.1016/01434160(90)90001-B.

33. Satoh H, Sperelakis N. Review of some actions of taurine on ion channels of cardiac muscle cells and others. Gen Pharmacol 1998; 30: 451-463, doi: 10.1016/S0306-3623(97) 00309-1.

34. Li F, Obrosova IG, Abatan O, Tian D, Larkin D, Stuenkel EL, et al. Taurine replacement attenuates hyperalgesia and abnormal calcium signaling in sensory neurons of STZ-D rats. Am J Physiol Endocrinol Metab 2005; 288: E29-E36, doi: 10.1152/ajpendo.00168.2004. 